\title{
Long-lasting, Specific Immunologic Unresponsiveness Associated with Cryptococcal Meningitis
}

\author{
David K. Henderson, John E. Bennett, and Margret A. Huber, Clinical \\ Center, Clinical Mycology Section, Laboratory of Clinical Investigation, \\ National Institute of Allergy and Infectious Diseases, National Institutes of \\ Health, Bethesda, Maryland 20205
}

A B S T R A C T A sensitive radioimmunoassay and an antibody class-specific enzyme-linked immunosorbent assay were used to determine whether patients cured of cryptococcosis responded normally to immunization with cryptococcal capsular polysaccharide (CPS) and type III pneumococcal polysaccharide. 10 normal volunteers and 8 patients who had been cured of cryptococcal meningitis and who had no serious underlying diseases were immunized with both antigens.

Geometric mean titers to CPS measured by radioimmunoassay were $1: 1$ in both groups before vaccination, but were 1:3 in patients and 1:119 in controls following immunization $(P<0.01$, Student's $t$ test $)$. Analysis of the class-specific response to immunization with CPS found little anti-CPS IgG or IgA. Geometric mean postvaccination IgM titers were 1:31 in patients and $1: 238$ in controls $(P<0.01)$. Responses to immunization with type III pneumococcal polysaccharide were similar in patients and controls, with $\operatorname{IgA}, \operatorname{IgM}$, and IgG mean titers of $1: 1129,1: 369$, and $1: 158$ in patients and $1: 1504,1: 1039$, and $1: 163$ in controls $(P>0.2$ for each antibody class).

Cured cryptococcal meningitis is often associated with prolonged specific immunologic unresponsiveness.

\section{INTRODUCTION}

Although conditions that cause defects in cellular immunity have been associated with an increased risk of developing cryptococcosis (1), the precise role of the humoral immune system in host defense against Cryptococcus neoformans remains conjectural. In 1974 Diamond and Bennett (2) reported that $60 \%$ of patients with cryptococcal meningitis did not have detectable levels of antibody against the type-specific capsular

Received for publication 27 October 1981 and in revised form 12 January 1982. polysaccharide of the yeast. This study, which used an indirect fluorescent assay for IgG antibody, also found that the absence of anticryptococcal antibody at the initiation of therapy was a significant risk factor for treatment failure.

The reason or reasons that patients with cryptococcal meningitis fail to produce antibody against cryptococcal polysaccharide $(\mathrm{CPS})^{1}$ remains uncertain. Such a trait might be inherited; however no outbreaks of cryptococcosis among kindred have been reported. Whereas investigators have found that injections of CPS induced a state of specific immunologic unresponsiveness in animals $(3,4)$, no studies have been conducted, to our knowledge, to determine whether absence of detectable antibody to CPS in sera of cryptococcosis patients is a result of specific immunologic unresponsiveness. The study to be reported here used a sensitive radioimmunoassay to address two different questions. First, do cured cryptococcosis patients have even less antibody to CPS than the normal population? Secondly, will these patients make a subnormal response when immunized with CPS?

\section{METHODS}

Normal volunteers. Sera from normal volunteers were obtained from Dr. John Robbins (Bureau of Biologics, Food and Drug Administration), from Dr. James C. Parke (University of North Carolina, Charlotte, NC), from Dr. Rafael Dolin (University of Vermont, Burlington, VT), from Dr. Paul Holland (Blood Bank, Clinical Center, National Institutes of Health), and from members of the Laboratory of Clinical Investigation. The age of these volunteers ranged from 2 to $72 \mathrm{yr}$ of age.

In a separate study, college age volunteers were hospitalized at the Clinical Center, and, after providing informed consent, were immunized with the polysaccharide vaccines to be described below.

\footnotetext{
${ }^{1}$ Abbreviations used in this paper: CPS, cryptococcal polysaccharide; ELISA, enzyme-linked immunosorbent assay; RIA, radioimmunoassay; SSSIII, type III pneumococcal polysaccharide.
} 
Cryptococcosis patients. Sera from 51 cryptococcosis patients were selected because the patients met the following criteria: (a) All had culture-proven cryptococcosis. (b) All patients were infected with serotype A C. neoformans. (c) Sera were available that had been obtained both before therapy had been administered and 1 yr following the completion of therapy. (d) All had been patients of the Clinical Mycology Section, Laboratory of Clinical Investigation so that the complete records were available on each patient.

In addition, eight patients who were still being actively followed at the Clinical Center following therapy for cryptococcal meningitis were included in the immunization studies. These patients met the following criteria: $(a)$ All had been cured of cryptococcal meningitis, and had been free of evidence of infection for a minimum of $1 \mathrm{yr}$ following completion of therapy for cryptococcal meningitis. (b) All had been free of cryptococcal antigen in both serum and cerebrospinal fluid for at least $1 \mathrm{yr}$ before immunization.

Other characteristics of the patients who were vaccinated were as follows. Their mean age was 46 yr (range, 32-70 $\mathrm{yr}$ ). An average of $5.5 \mathrm{yr}$ (range 1.5-11.5 yr) had elapsed since completion of therapy. All eight of these patients had cryptococcal antigen in CSF and four of eight had cryptococcal antigen in serum at the time of diagnosis as measured by the latex agglutination test (5). Antigen was not detectable in these patients, in either serum or CSF, for an average of $3.4 \mathrm{yr}$ (range, 1.1-8.6 yr) at the time of CPS injection. One of the patients had serum antibody at the time of diagnosis and one patient developed antibody during treatment for his infection (titers $1: 2$ and $1: 1$, respectively). The remaining six patients had never produced detectable antibody in response to their infections. All eight patients were in good health. None had hematologic or reticuloendothelial malignancies, and none were receiving immunosuppressive chemotherapy.

Radioimmunoassay (RIA). Type A CPS was prepared from culture supernatant (6) and contained $0.17 \% \mathrm{~N}$ by microkjeldahl distillation and nesslerization. CPS was activated with $\mathrm{CNBr}$ using $\mathrm{pH} 11.0$ and a weight ratio of CPS:CNBr of 5:1. Tyraminylation was carried out at pH 8.5. Free tyramine was removed by dialysis against $400 \mathrm{vol}$ of toluene saturated phosphate-buffered saline with four changes over $4 \mathrm{~d}$. Tyramine content of the tyraminyl-CPS was measured by Lowry (7) and CPS was measured by anthrone reagent (8) using a CPS standard. Assuming molar ratios of hexose:pentose of 2:1 (9), we calculated that one tyramine group was introduced per 139 monosaccharide units. After iodination with ${ }^{125} \mathrm{I}$ and chloramine- $T$, the ${ }^{125}$ I-tyraminyl CPS ( ${ }^{125} \mathrm{I}-$ tyr-CPS) was dialyzed against four changes of $\mathrm{NaCl}$ $(0.15 \mathrm{M})$, diluted with phosphate buffer $(0.01 \mathrm{M}, \mathrm{pH} 7.4)$, and chromatographed on DEAE cellulose (Whatman DE 52, Reeve, Angel Company, Clifton, NJ) that had been equilibrated with the same buffer. CPS was eluted with $0.2 \mathrm{M}$ $\mathrm{NaCl}$ in $0.01 \mathrm{M}$ phosphate buffer (pH 7.4). Specific activity was calculated to be $2.0 \times 10^{7} \mathrm{cpm} / \mu \mathrm{g}$ CPS, assuming complete recovery of polysaccharide from the DEAE column. All samples in the study were counted in a gamma spectrometer (model 1185, Nuclear Chicago Corp., Chicago, IL) having an efficiency of $86 \%$ for ${ }^{125} \mathrm{I}$. Assuming an average CPS molecular weight of one million (9), $\sim 2 \mathrm{~mol}$ of ${ }^{125} \mathrm{I}$ were introduced per mole of CPS. Approximately $95 \%$ of the radioactivity could be precipitated by high titer antibody in the assay.

RIA for antibody to CPS was performed using the Farr test (10). Using $20 \%$ binding as the lower limit of positivity, this assay is sensitive to $136 \mathrm{ng} / \mathrm{ml}$ of anti-CPS antibody.

In the vaccination experiments, results were compared using log base 2 antibody titers. A least-squares line of bestfit was obtained for percent bound as a function of log dilution. The intercept of $20 \%$ bound and the least-squares line was used as the titer.

Enzyme linked immunosorbent assay (ELISA). ELISA were used to detect the class-specific antibody response to immunization with both CPS and type III pneumococcal polysaccharide (SSSIII). The procedures used were modifications of the original ELISA procedures described by Engvall and Perlmann (11). Wells in polystyrene microtiter substrate plates (Dynatech Laboratories, Alexandria, VA) were coated with $100 \mu \mathrm{l}$ of solution containing either 12 $\mu \mathrm{g} / \mathrm{ml}$ of CPS or $7 \mu \mathrm{g} / \mathrm{ml}$ of SSSIII in $0.05 \mathrm{M}$ carbonate buffer (pH 9.6) for $18 \mathrm{~h}$ at $37^{\circ} \mathrm{C}$. Serum dilutions were incubated in duplicate for $2 \mathrm{~h}$ at $37^{\circ} \mathrm{C}$ in antigen-coated wells.

Class-specific (IgA, IgG, and IgM) goat anti-human immunoglobulin that was conjugated to horseradish peroxidase (Kirkegaard and Perry Laboratories, Gaithersburg, MD) was diluted in $0.45 \%$ sodium chloride, $3 \%$ bovine serum albumin (Pentex, Miles Research Laboratories, Elkhart, IN), and 0.05\% Tween 20 (Sigma Chemical Co., St. Louis, MO).

$O$-phenylenediamine was used as substrate (Aldrich Chemicals, Milwaukee, WI). Reactions were stopped by the addition of $20 \mu$ l of $8 \mathrm{~N}$ sulfuric acid. Absorption was measured as $492 \mathrm{~nm}$ in an MR580 ELISA reader (Dynatech Laboratories). As a control each plate contained three dilutions in duplicate of a standard serum. Optical density was plotted as a function of $\log$ serum dilution for a minimum of six dilutions of each serum, and a best-fit line constructed by least-squares, using the linear part of the slope. The optical density of a specified dilution of the standard serum was derived from the curve of that serum. Then, the intercept of this optical density with curves of the unknown sera was used to determine titer. By convention, any serum that was negative in the lowest dilution tested (1:4) was assigned a titer of 2

Quantitative determination of IgM levels. Postvaccination serum samples from each of the patients and volunteers who were immunized in the study were evaluated for quantitative levels of IgM by immunofluorescence (ImmunofluorIgM, Bio-Rad Laboratories, Richmond, CA) using a photofluorometer (American Instrument Co., Silver Spring, MD).

Vaccination studies. Serotype A C. neoformans CPS was obtained from Dr. K. J. Kwon-Chung, National Institute of Arthritis and Infectious Diseases (NIAID). This material had been purified as previously described (6) from culture supernatants, and contained $0.7 \%$ nitrogen by microkjeldahl analysis. CPS was suspended in sterile water, filtered with a $0.45-\mu \mathrm{m}$ Millipore filter (Millipore Corp., Bedford, MA) and dispensed into sterile vials. SSSIII was produced by Eli Lilly \& Co., Indianapolis, IN and obtained from Dr. James Hill, NIAID. The SSSIII preparation had been found to contain $0.2 \%$ nitrogen and $2 \%$ nucleic acid. After informed consent had been obtained, volunteers and patients were vaccinated with $0.5 \mathrm{ml}$ of each preparation (37 $\mu \mathrm{g}$ of CPS; 50 $\mu \mathrm{g}$ of SSSIII)

Studies were conducted to determine the effect of vaccination with one polysaccharide on the antibody response to the other. Volunteers were vaccinated with either CPS or SSSIII then 2 wk later with the remaining antigen. Antibody responses were measured by RIA and ELISA and compared with those obtained when both antigens were administered simultaneously. No cross-reactions or antigenic competition between the two vaccines were found. Additionally, in other pilot studies, volunteers were vaccinated with various doses of CPS (doses used: $3.7,7.4,18.5,37$, and $74 \mu \mathrm{g}$ ). The optimal response to immunization occurred at the $37-\mu \mathrm{g}$ dose of CPS. 
Thus, for studies in which the normal volunteer response to immunization was compared with the response of patients who had been cured of cryptococcal meningitis, both the patients and the volunteers were immunized with $37 \mu \mathrm{g}$ of CPS and $50 \mu \mathrm{g}$ of SSSIII on the same day.

\section{RESULTS}

Prevalence of anti-CPS antibody in normal volunteers. $89 \%$ of the 185 serum samples obtained from normal volunteers demonstrated binding of CPS. The magnitude of the binding in these samples, however, was small. When three of the sera which, when undiluted, demonstrated nearly $100 \%$ binding of available antigen, were diluted 1:10, the binding in each serum fell to $<50 \%$. A small but statistically significant decrease $(P<0.05$, Wilcoxon test $)$ in the prevalence of antibody was noted with increasing age (Table I).

Prevalence of anti-CPS antibody in patients with cured cryptococcal meningitis. Only 21 of the 51 patients evaluated $(41 \%)$ had detectable antibody against CPS on at least one occasion by RIA (Table I). The trend noted in the normal volunteers of decreasing prevalence of anti-CPS antibody with increasing age was also noted in the cryptococcosis patients. The prevalence of antibody in the entire group of patients was significantly different from the overall prevalence in the volunteer studies $(41 \%$ vs. $89 \%)(P<0.002$, chisquare).

Immunization studies. When the patients' and volunteers' serologic responses to injection with CPS were measured by RIA, both the patients and the volunteers demonstrated significant increases in titers $(P$

TABLE I

Prevalence of Anticryptococcal Antibody in Normal Volunteers and Cryptococcosis Patients

\begin{tabular}{|c|c|c|}
\hline & Volunteers & Patients \\
\hline Age & $\frac{\text { Positive }^{\bullet}}{\text { Tested }^{\circ}}$ & $\frac{\text { Positive }^{*}}{\text { Tested }^{-}}$ \\
\hline$y r$ & $\%$ & $\%$ \\
\hline $2-17$ & $\frac{88}{95}(93)$ & $\frac{2}{3}(67)$ \\
\hline $18-33$ & $\frac{36}{41}(88)$ & $\frac{6}{12}(50)$ \\
\hline $34-49$ & $\frac{7}{8}(88)$ & $\frac{4}{14}(29)$ \\
\hline $50-74$ & $\frac{33}{41}(81)$ & $\frac{9}{22}(41)$ \\
\hline Total & $\frac{164}{185}(89)$ & $\frac{21}{51}(41)$ \\
\hline
\end{tabular}

- Positive sera defined as those undiluted sera that bind $\geq 20 \%$ of available antigen in RIA.

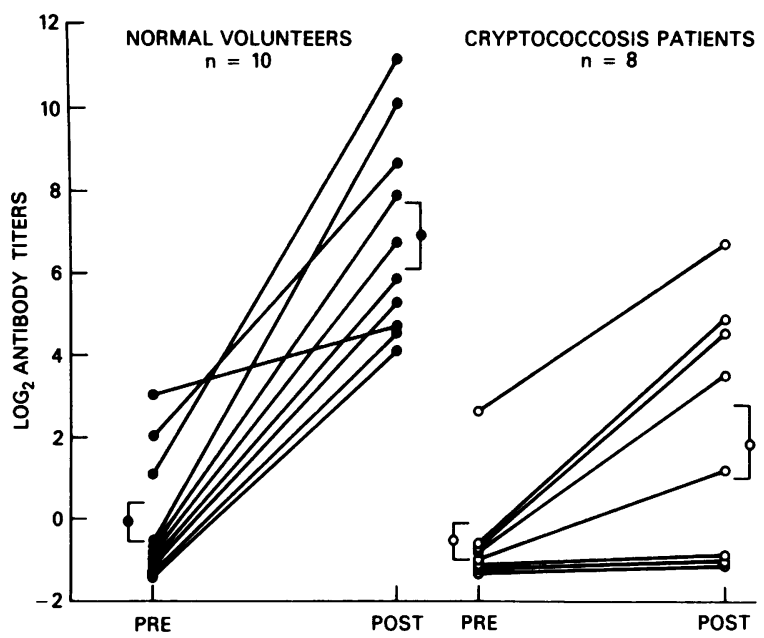

Figure 1 Responses of normal volunteers and cryptococcosis patients to immunization with CPS as measured by RIA. Post samples were obtained 2 wk following immunization. Each line represents an individual patient; the summary points represent the mean value plus and minus the standard error of the mean for each group before and after immunization. By convention, any serum sample that bound less than $20 \%$ of ${ }^{125}$ I-tyraminyl-CPS was assigned a titer of 0.5 .

$<0.01$ for patients; $P<0.001$ for volunteers: onetailed, paired Student's $t$ test) (Fig. 1). Both the normal volunteers and cured patients had geometric mean prevaccination titers of 1:1. Geometric mean anti-CPS antibody titers $2 \mathrm{wk}$ following vaccination, however, were significantly different between the two groups. In the group of cryptococcosis patients, the geometric mean titer after CPS immunization was 1:3 (Fig. 1, right), whereas in the group of normal volunteers, the geometric mean postvaccination titer was 1:119 (Fig. 1 , left). This difference was statistically significant $(P$ $<0.01$, one-tailed Student's $t$ test). All 10 of the volunteers responded by elevating anti-CPS antibody titers, whereas only 5 of the 8 patients demonstrated any measurable rise in anti-CPS antibodies. 7 of 10 volunteers, and 1 of 8 patients developed postvaccination antibody titers of $1: 32$ or greater.

When the two groups were compared by ELISA for the class-specific response to immunization with CPS, the major response to immunization was found to be in the IgM class. Only the volunteers demonstrated a statistically significant increase in IgM anti-CPS antibody (Fig. 2) $(P<0.001$ by the paired, one-tailed Student's $t$ test). The volunteers' geometric mean prevaccination titer was $1: 73$ and the postvaccination titer was 1:238. In the group of patients, however, the geometric mean prevaccination titer was $1: 38$ and the postvaccination titer was $1: 31$. 9 of 10 volunteers responded to immunization with CPS by elevating IgM anti-CPS antibodies, whereas only 2 of 8 patients de- 


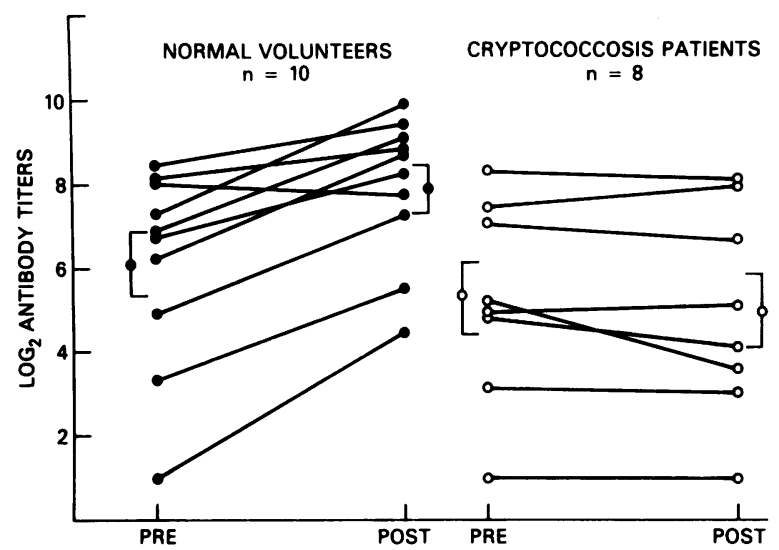

Figure 2 IgM responses of normal volunteers and cryptococcosis patients to immmunization with CPS as measured by ELISA. Post samples were obtained 2 wk following immunization. Each line represents an individual patient; the summary points represent the mean value plus and minus the standard error of the mean for each group before and after immunization.

veloped modest elevations in IgM anti-CPS titers. The difference in IgM anti-CPS response between the patients and volunteers was again statistically significant $(P<0.01$ by the one-tailed Student's $t$ test). Neither patients nor volunteers demonstrated statistically significant elevations in either IgA or IgG titers to CPS when pre- and postvaccination samples were compared, nor was the geometric mean response different between the two groups.

Thus, patients cured of systemic cryptococcal infection, who had been free of CPS in serum and CSF for at least $1 \mathrm{yr}$ responded poorly to immunization with CPS, whereas normal volunteers demonstrated a significantly greater response. A minor response was seen in five of the eight patients (Fig. 1). Of the five, only one had had serum antigen detected during infection. Thus, patients who have antigen in serum at the time of diagnosis may be more likely to demonstrate this immunologic unresponsiveness. The one patient who developed an antibody titer greater than 1:32 (Fig. 1) was also the only patient we studied who developed anti-CPS antibody during the course of his primary infection. Moderate titers of anti-CPS antibodies have persisted in his serum since his infection. He was also one of the two patients who, following immunization, developed elevated IgM anti-CPS antibodies as measured by ELISA (Fig. 2).

With respect to the response to immunization with SSSIII, both the patients and the controls responded similarly. Both the patients and the controls significantly elevated IgA, IgG, and IgM titers against SSSIII (Fig. 3). The titers in the cryptococcosis patients after immunization were not significantly different from the normal volunteers $(P>0.2$ by the paired, one-tailed Student's $t$ test for each antibody class). Similarly, the preimmunization titers and fold rises of the two groups did not differ significantly.

Because of the apparent difference in IgM response to CPS, we studied the postimmunization sera for quantitative IgM levels. Mean values for the two groups were as follows: Volunteers: mean value $169.3 \pm 19.8 \mathrm{mg} / 100 \mathrm{ml}$, range $86-286 \mathrm{mg} / 100 \mathrm{ml}$; patients: mean value $143.4 \pm 17 \mathrm{mg} / 100 \mathrm{ml}$, range 70 $221 \mathrm{mg} / 100 \mathrm{ml}(P>0.4$ when compared by one-tailed Student's $t$ test). No individual patient or volunteer had a value that fell below the normal range (normal for this test: $60-200 \mathrm{mg} / 100 \mathrm{ml}$ ).

\section{DISCUSSION}

Anti-CPS antibodies were detected in $88.6 \%$ of normal volunteers, though only in very low titers, represented by percent bound values between 21.2 and $89 \%$. That the RIA measured binding by nonimmunoglobulin proteins appears unlikely. RIA on fractions obtained with one normal serum after Sephacryl chromatography or sucrose density gradient ultracentrifugation indicated molecular weights of the binding protein compatible with immunoglobulin (data not shown). Addition of calcium chelators did not alter binding, suggesting that $\mathrm{C}$-reactive protein was not responsible (data not shown). The immunizing stimulus for these antibodies is unknown. Such antibodies may arise as a result of exposure to $C$. neoformans in nature (12, 13), or may have arisen by exposure to different immunologic stimuli that provoke cross-reacting anti-

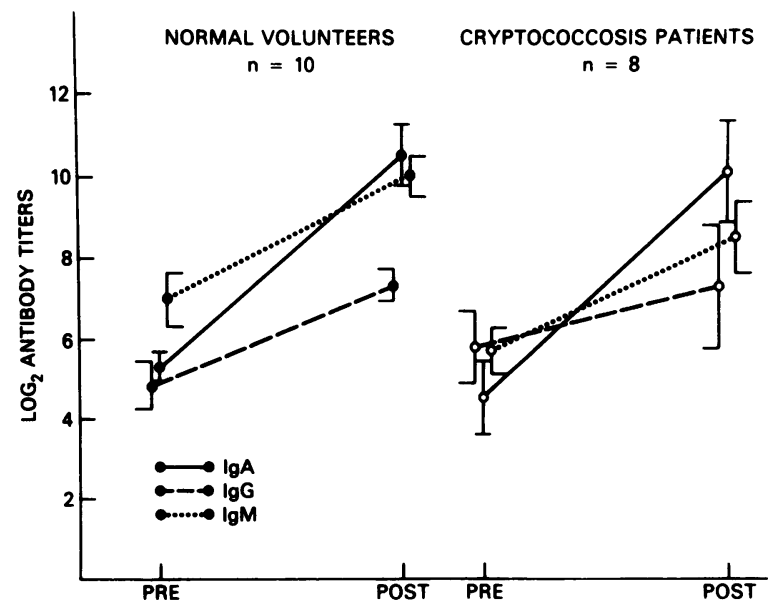

Figure 3 IgM, IgA, and IgG responses of normal volunteers and cryptococcosis patients to immunization with SSSIII measured by ELISA 2 wk after immunization. Lines represent the mean plus and minus the standard error of the mean for each group before and after immunization. 
bodies $(6,14,15)$. Similar experience has been encountered with normal humans having antibody to dextran (16), to melibiose (17), and to certain microbial polysaccharides (18).

In the group of cured cryptococcosis patients the prevalence of anti-CPS antibody as measured by RIA was $41.1 \%$ (Table $\mathrm{I}$ ). This prevalence is similar to that encountered with other sensitive methods $(2,19-21)$, and is significantly lower than the control population studied by RIA $(P<0.002$, chi-square).

Because of the reports of decreasing immunoglobulin levels with increasing age (22-24) and because of evidence that low preimmunization IgM and IgA levels in aged individuals correlated with blunted response to pneumococcal vaccination (25), we determined total serum IgM levels in both the patients and the volunteers. Whereas there was no difference detected when the two groups were compared using the Student's $t$ test $(P>0.4)$, the type II error is large because of the small numbers of samples in each group. No patient, however, was detected as having a serum IgM value below the normal value for the test that was used.

Three distinct possibilities exist for the diminished prevalence of antibody in the patient population. First, the ability to mount an antibody response to infection with C. neoformans or to vaccination with CPS may be genetically determined. Thus, a subpopulation of patients who develop cryptococcal infection may be genetic hyporesponders. Human antibody response to bacterial flagellin (26), and polysaccharides of Haemophilus influenzae $(27,28)$, meningococcus group C (27), and Streptococcus pneumoniae (28) have been linked to immunoglobulin allotype. Such studies have not been conducted on patients with cryptococcosis. Alternately, patients who develop systemic infection with $C$. neoformans may have their antibody responses masked by excess CPS present in their body fluids. Although CPS may persist in the infected host for an undefined amount of time, the immunization studies carried out up to $8 \mathrm{yr}$ after antigen had no longer been detectable in serum or CSF, make this explanation seem less likely. A final possibility is that patients who develop systemic cryptococcal infection may, during the course of their illness, develop a state of immunologic unresponsiveness.

Immunization of eight cryptococcosis patients with CPS produced a response that was heterogeneous, but blunted in comparison to the antibody response of normal volunteers. The two groups did not differ significantly with respect to their responses to immunization with SSSIII. To our knowledge, only one previous study, that of Norden and his colleagues (29), has documented that a prior infection can diminish antibody response to antigens from the same microbe. In their study, children who had had $H$. influenzae meningitis and orbital cellulitis failed to respond to immunization with the capsular polysaccharide of the infecting organism. This earlier study does not address the issue of the specificity of the immunologic unresponsiveness in these children with $H$. influenzae infections. In addition, although it seems likely that their patients had antigenemia previously, this was not documented.

The results of our study support the concept that long-term specific unresponsiveness to challenge with CPS also exists in humans in association with cryptococcosis.

\section{REFERENCES}

1. Lewis, J. L., and S. Rabinovich. 1972. The wide spectrum of cryptococcal infections. Am. J. Med. 53: 315-322.

2. Diamond, R. A., and J. E. Bennett. 1974. Prognostic factors in cryptococcal meningitis. A study in 111 cases. Ann. Intern. Med. 80: 176-181.

3. Murphy, J. W., and G. C. Cozad. 1972. Immunological unresponsiveness induced by cryptococcal capsular polysaccharide assayed by the hemolytic plaque technique. Infect. Immun. 5: 896-901.

4. Kozel, T. R., W. F. Gulley, and J. Cazin, Jr. 1977. Immune response to Cryptococcus neoformans soluble polysaccharide: immunological unresponsiveness. Infect. Immun. 18: 701-707.

5. Bloomfield, N., M. A. Gordon, and D. F. Elmendorf, Jr. 1963. Detection of Cryptococcus neoformans antigen in body fluids by latex particle agglutination. Proc. Soc. Exp. Biol. Med. 114: 64-67.

6. Bennett, J. E., and H. F. Hasenclever. 1965. Cryptococcus neoformans polysaccharide: Studies of serologic properties and role in infection. J. Immunol. 94: 916920.

7. Lowry, O. H., R. J. Rosebrough, A. L. Farr, and R. J. Randall. 1951. Protein measurement with the Folin phenol reagent. J. Biol. Chem. 193: 265-275.

8. Dische, Z. 1960 . New color reactions for the determination of sugars in polysaccharides. In Methods of Biochemical Analysis. D. Glick, editor. Wiley Interscience Publishers Inc., New York. 2: 313-358.

9. Merrifield, E. H., and A. M. Stephen. 1980. Structural investigations of two capsular polysaccharides from Cryptococcus neoformans. Carbohydr. Res. 86: 69-76.

10. Farr, R. S. 1958. A quantitative immunochemical measure of the primary interaction between $I^{\circ} \mathrm{BSA}$ and antibody. J. Infect. Dis. 103: 239-262.

11. Engvall, E., and P. Perlman. 1971. Enzyme-linked immunosorbent assay (ELISA). Quantitative assay of immunoglobulin G. Immunochemistry. 8: 871-874.

12. Powell, K. E., B. A. Dahl, R. J. Weeks, and F. E. Tosh. 1972. Airborne Cryptococcus neoformans: Particles from pigeon excreta compatible with alveolar deposition. J. Infect. Dis. 125: 412-415.

13. Neilson, J. B., R. A. Fromtling, and G. S. Bulmer. 1977. Cryptococcus neoformans: Size range of infectious particles from aerosolized soil. Infect. Immun. 17: 634-638.

14. Evans, E. E., L. J. Sorensen, and K. W. Walls. 1953. The antigenic composition of Cryptococcus neoformans. $\mathrm{V}$. A survey of cross-reactions among strains of cryptococcus and other antigens. J. Bacteriol. 66: 287-293. 
15. Rebers, P. A., S. A. Barker, M. Heidelberger, Z. Dische, and E. E. Evans. 1958. Precipitation of the specific polysaccharide of Cryptococcus neoformans type A by types II and XIV antipneumococcal sera. J. Am. Chem. Soc. 80: 1135-1137.

16. Palosuo, T., and F. Milgrom. 1981. Appearance of dextrans and antidextran antibodies in human sera. Int. Arch. Allergy Appl. Immun. 65: 153-161.

17. Bird, G. W. G., and T. C. F. Roy. 1980. Human serum antibodies to melibiose and other carbohydrates. Vox Sang. 38: 169-171.

18. Hoff, G. E., and N. Hoiby. 1978. Precipitating antibodies against Neisseria meningitidis in normal sera and their possible origin. Acta Pathol. Microbiol. Scand. Sect. C. 86: 123-129.

19. Gordon, M. A., and D. K. Vedder. 1966. Serologic tests in diagnosis and prognosis of cryptococcosis. JAMA (J. Am. Med. Assoc.). 197: 961-967.

20. Bindschadler, D. D., and J. E. Bennett. 1968. Serology of human cryptococcosis. Ann. Intern. Med. 69: 45-52.

21. Kimball, H. R., H. F. Hasenclever, and S. M. Wolff. 1967. Detection of circulating antibody in human cryptococcosis by means of a bentonite flocculation technique. Am. Rev. Respir. Dis. 95: 631-637.

22. Buckley, C. E., III, and F. C. Dorsey. 1970. The effect of aging on human serum immunoglobulin concentrations. J. Immunol. 105: 964-972.

23. Buckley, C. E., III, and F. C. Dorsey. 1971. Serum immunoglobulin levels throughout the life-span of healthy man. Ann. Intern. Med. 75: 673-682.
24. Schwick, H. G., and W. Becker. 1969. Humoral antibodies in older humans. In Current Problems in Immunology. O. Westphal, H. E. Beck and F. Brundmann, editors. Springer Verlag, Berlin. 253-257.

25. Amann, A. J., G. Schiffman, and R. Austrian. 1980. The antibody responses to pneumococcal capsular polysaccharides in aged individuals. Proc. Soc. Exp. Biol. Med. 164: 312-316.

26. Whittingham, S., J. D. Mathews, M. S. Schanfield, J. V. Matthews, B. D. Tait, P. J. Morris, and I. R. MacKay. 1980. Interactive effect of Gm allotypes and HLA-B locus antigens on the human antibody response to a bacterial antigen. Clin. Exp. Immunol. 40: 8-15.

27. Pandey, J. P., G. Virella, C. B. Loadholt, H. H. Fudenberg, C. U. Kyong, R. M. Galbraith, E. C. Gotschlich, and J. C. Parke, Jr. 1979. Association between immunoglobulin allotypes and immune response to Haemophilus influenzae and meningococcus polysaccharides. Lancet. I: 190-192.

28. Siber, G. R., P. H. Schur, A. C. Aisenberg, S. A. Weitzman, and G. Schiffman. 1980. Correlation between serum IgG-2 concentrations and the antibody response to bacterial polysaccharide antigens. N. Engl. J. Med. 303: 178-182.

29. Norden, C. W., R. H. Michaels, and M. Melish. 1975. Effect of previous infection on antibody response of children to vaccination capsular polysaccharide of $\mathrm{He}$ mophilus influenzae type b. J. Infect. Dis. 132: 69-74. 